\title{
Combination of IL-24 and cisplatin inhibits angiogenesis and lymphangiogenesis of cervical cancer xenografts in a nude mouse model by inhibiting VEGF, VEGF-C and PDGF-B
}

\author{
ZHAOXIA WANG ${ }^{1}$, JIYUAN LV ${ }^{1}$ and TIANTIAN ZHANG ${ }^{2}$ \\ ${ }^{1}$ The First Hospital of Shanxi Medical University, Taiyuan, Shanxi; ${ }^{2}$ Linfen City People's Hospital, Linfen, Shanxi, P.R. China
}

Received November 18, 2014; Accepted February 24, 2015

DOI: $10.3892 /$ or.2015.3853

\begin{abstract}
The aim of the present study was to investigate the synergistic inhibitory effects of recombinant IL-24 delivered by pDC316-hIL-24 transfection and cisplatin (DDP) on angiogenesis and lymphangiogenesis in a cervical cancer xenograft model established in nude mice. Thirty-six mice (successful model) were randomly divided into six groups ( $n=6$ in each): i) phosphate-buffered saline control; ii) empty plasmid; iii) half-dose DDP; iv) recombinant interleukin (IL)-24; v) full-dose DDP; and vi) combined treatment. Tumor growth and animal weight were measured every 3 days. Animals were sacrificed by cervical dislocation at 2 weeks after the cessation of treatment. Tumor inhibition was compared after intervention. Lymph node metastasis was evaluated by immunohistochemical (IHC) analysis of vascular endothelial growth factor (VEGF), VEGF-C and VEGFR-3. Platelet-derived growth factor (PDGF)-B expression was also investigated by western blot analysis. Microvessel density was evaluated by IHC analysis of CD34 expression. The tumor growth was slower or reduced in the IL-24 and half-dose DDP+IL-24 groups. The expression of VEGF and microvessel density in the IL-24 group was significantly lower than that in the other groups. VEGF (VEGF-A), VEGF-C, VEGFR-3 and PDGF-B expression was significantly decreased in the IL-24 and halfdose DDP+IL-24 groups compared with that in the other groups $(\mathrm{P}<0.001)$. The recombinant plasmid pDC316-hIL-24 acts synergistically with cisplatin to inhibit tumor growth and angiogenesis. Our data indicate that these effects are mediated by downregulation of VEGF, VEGF-C and PDGF-B expression. Thus, IL-24 may enhance tumor chemosensitivity to cisplatin, which may be an important strategy for reducing the side-effects of this chemotherapy.
\end{abstract}

Correspondence to: Dr Jiyuan Lv, The First Hospital of Shanxi Medical University, Taiyuan, Shanxi, P.R. China

E-mail: jiyuanlvsci@126.com

Key words: IL-24, cervical cancer, cisplatin, VEGF, VEGFR-3, PDGF-B

\section{Introduction}

Cervical cancer is associated with high incidence and mortality. It is the second most common malignancy worldwide among females, and is the seventh most common cancer overall, with more than 527,000 new cases diagnosed in 2012 (8\% of female cases and $4 \%$ of the total). Standard treatment includes surgical treatment, radiotherapy and chemotherapy. The sideeffects of chemotherapy are adverse, such as cisplatin, which is associated with serious side-effects and the development of resistance; therefore, additional strategies for the treatment of cervical cancer are urgently required (1).

Tumor angiogenesis plays an important role in the processes of tumor growth, invasion and metastasis. Platelet-derived growth factor (PDGF) and other growth factors, particularly vascular endothelial growth factor (VEGF), promote tumor angiogenesis and tumor invasion through autocrine and/or paracrine mechanisms. In pancreatic cancer cell lines, PDGF-B specifically binds to the receptor, PDGFR- $\beta$, to activate the Notch-1 and NF- $\mathrm{B}$ signaling pathway. This results in increased expression of VEGF, which promotes tumor angiogenesis (2). Thus, downregulation of PDGF-B expression may effectively inhibit the proangiogenic effects of VEGF.

Interleukin (IL)-24, also known as melanoma differentiation-associated gene-7, has broad-spectrum antitumor activity in a variety of tumors such as lung (3), ovarian (4) and breast cancers (5). It has been shown to exert significant growth inhibition and apoptosis induction effects, with no obvious adverse effects on normal cells (6).

Our preliminary studies showed that delivery of exogenous IL-24 using the cationic liposome-mediated method for transfection with the recombinant plasmid pDC316IL-24 significantly augmented the sensitivity of tumors to cisplatin in a nude mouse cervical cancer xenograft model (7). However, the mechanism underlying this effect remains to be elucidated.

In the present study, the synergistic inhibitory effects of the recombinant plasmid pDC316-hIL-24 and cisplatin on angiogenesis and lymphangiogenesis were investigated in a cervical cancer xenograft model established in nude mice. Furthermore, the effects on the expression of VEGF-C/VEGFR-3 and PDGF-B and microvessel density in the cervical cancer xenografts were investigated to clarify the mechanism. This 
information will be important in developing improved strategies for the treatment of cervical cancer.

\section{Materials and methods}

Cell culture. Human cervical carcinoma HeLa cells were provided by the Cell Center of the Union Medical College (Beijing, China). Cells were cultured in Dulbecco's modified Eagle's medium (DMEM) high glucose-complete medium at $37^{\circ} \mathrm{C}$ in a $5 \% \mathrm{CO}_{2}$ incubator. Logarithmic growth phase cells were collected, and the cell number was adjusted to $1 \times 10^{7} / \mathrm{ml}$.

Use and care of experimental animals. Specific pathogen-free (SPF) female nude mice (BALB/c; 13-15 g, aged 4 weeks) were obtained from the Shanghai Silaike Experimental Animal Co., Ltd. (Shanghai, China). Mice were maintained in pathogen-free conditions and used for study in accordance with the National Institutes of Health Guide for the Care and Use of Laboratory Animals. All surgical procedures and care administered to the animals were approved by the Institutional Ethics Committee.

Ethics statement. All surgical procedures and care administered to the animals were approved by the Institutional Ethics Committee.

Evaluation of the benefits for the experimental animals. i) The experiment, in which the needs of the animals were fully considered, was approved by the ethics committee, including physiological (adequate food, water, temperature and illumination), environmental, psychological and social needs (socially raised, 4-6 animals per cage, avoid tiredness and overstimulation). The outcomes of the preliminary experiment and the literature were taken into consideration to make rational design of the sample size and operation standard. ii) A daily observation was preformed to prevent the animals from anger, comfortlessness, fear, nervousness, pain or damage and to keep them at normal status. Abuse, excessive or incorrect medication was avoided. For subcutaneous injection, which was easy to operate, narcotics were not applicable; for tail vein injection, which was not easy to operate, intraperitoneal anesthesia was given to alleviate the pain of the animals. iii) At the time of endpoint, the animals were sacrificed within $15 \mathrm{sec}$ to avoid the nervousness of the other animals.

Xenograft animal model and grouping. The xenograft tumor model was established by subcutaneous (s.c.) injection of $\sim 1 \times 10^{6}$ HeLa cells (100 $\mu 1$ suspension) into the left axillary lateral of each nude mouse. The model was deemed to be established successfully following the appearance of xenografted tumors after 7 days and with tumor diameters of $\sim 4-5 \mathrm{~mm}$ after 14 days. Subsequently, successfully xenografted mice were randomly divided into six groups $(n=6$ per group): i) PBS buffer control group: $100 \mu 1$ injected once per 3 days for a total of five times; ii) empty plasmid group: empty plasmid pDC316 (Benyuan Zhengyang Gene Technology Co., Beijing, China); $100 \mathrm{mg} / \mathrm{l}, 100 \mu \mathrm{l}$ once per 3 days, for a total of five times) and liposome Lipofectamine 2000 (Invitrogen, Shanghai, China; $25 \mu$ l for transfection); iii) half-dose DDP (cisplatin) group: intraperitoneal (i.p.) injection of cisplatin (Qilu Pharmaceutical Co., Ltd., Jinan, China; $2.5 \mathrm{mg} / \mathrm{kg}$,
$100 \mu \mathrm{l}$ once a day for 3 days); iv) IL-24 group: local multipoint intratumoral injection of pDC316-hIL-24 (Benyuan Zhengyang Gene Technology Co.); $100 \mathrm{mg} / 1,100 \mu 1$ once per 3 days for a total of five times) and liposome Lipofectamine 2000 (25 $\mu \mathrm{l}$ for transfection); v) full-dose DDP (cisplatin) group (DDP group): i.p. injection of cisplatin $(5 \mathrm{mg} / \mathrm{kg}, 100 \mu \mathrm{l}$ i.p. once a day for a total of 3 days; and vi) combined treatment group: cisplatin $(2.5 \mathrm{mg} / \mathrm{kg}, 100 \mu \mathrm{l}$ i.p. once a day for 3 days) followed by local multipoint intratumoral injection of pDC316-hIL-24 (100 mg/l, $100 \mu 1$ once per 3 days for a total of five times). The tumor size (L) and short diameter (W) were measured every 3 days with a vernier caliper. Weight and the overall status of the mice were recorded at the same time. Nude mice were sacrificed by cervical dislocation at 2 weeks after the cessation of treatment. Tumor volume was measured and weighed; and the inhibition of tumor weight was calculated. Tumor size $\mathrm{V}\left(\mathrm{mm}^{3}\right)=\mathrm{L} \times \mathrm{W}^{2} / 2$.

RT-PCR analysis of IL-24 expression. Determination of pDC316-hIL-24 expression in the xenografted tumors was performed using PrimeScript ${ }^{\mathrm{TM}}$ First Strand cDNA synthesis kit (Takara Biotechnology, Dalian, China). Primers of IL-24 were: forward, 5'-GCCAAGCTTATGAATTTTCAACAGA GG-3' and reverse, 5'-GCCGTCGACCTAGAGCTTGTAGAA TTT-3'. Expression of GADPH was analyzed as a control using the following primers: forward, 5'-TGAACGGGAAGCTC ACTGG-3' and reverse, 5'-TCCACCACCCTGTTGCTGGA-3'. The amplification was performed using the Applied Biosystems GeneAmp PCR system 2700 with the following reaction conditions: a $94^{\circ} \mathrm{C}$ pre-denaturation for $2 \mathrm{~min}$, followed by 32 cycles of a $94^{\circ} \mathrm{C}$ denaturation for $20 \mathrm{sec}$, a $58^{\circ} \mathrm{C}$ annealing for $20 \mathrm{sec}$ and $\mathrm{a} 72^{\circ} \mathrm{C}$ extension for $20 \mathrm{sec}$, with a final extension at $72^{\circ} \mathrm{C}$ for $8 \mathrm{~min}$. PCR products were separated by $1.5 \%$ agarose gel electrophoresis.

Immunohistochemical detection of VEGF, VEGF-C, VEGFR-3 and P-CK. After dewaxing, hydration and antigen retrieval, VEGF expression was detected by immunohistochemical staining with primary and secondary detection antibodies (Boster, Wuhan, China). Immunoreactivity was visualized by incubation with DAB chromogenic agent DAB kit (Zhongshan Jinqiao Biological Engineering Co., Beijing, China). Staining was evaluated in 10 randomly selected fields per section, with intensity divided into four levels as follows: 0 , no staining; 1 , pale yellow; 2 , brown; and 3, tan. Scores were assigned according to the percentage of positive cells in each field of vision as follows: 10\%, 0 points; 11-20\%, 1 point; $21-60 \%, 2$ points; and $61-100 \%, 3$ points. The average staining intensity score (IS) was calculated for each section based on Bresalier's semiquantitative method (8). IS represents the average multiplied product of the staining intensity score and the positive cell score per 10 randomly selected fields of vision in each section.

Immunohistochemical (IHC) detection of CD34 for microvessel density (MVD) enumeration. Solid tumor growth and metastasis require sustained angiogenesis, the extent of which is indicated by MVD. Microvessel enumeration can be achieved by IHC labeling of CD34, which is expressed stably and specifically by tumor capillaries and endothelial cells (9). 
Table I. Weight change of nude mice $(\mathrm{g})$ and tumor volume change $\left(\mathrm{mm}^{3}\right)$ after intervention $(\mathrm{mean} \pm \mathrm{SD})$.

\begin{tabular}{|c|c|c|c|c|c|c|}
\hline $\begin{array}{l}\text { After } \\
\text { therapy (days) }\end{array}$ & $\begin{array}{l}\text { Control } \\
\text { group }\end{array}$ & $\begin{array}{c}\text { Empty } \\
\text { plasmid group }\end{array}$ & $\begin{array}{l}\text { Half-dose } \\
\text { DDP group }\end{array}$ & IL-24 group & $\begin{array}{l}\text { Full-dose } \\
\text { DDP group }\end{array}$ & $\begin{array}{c}\text { Half-dose } \\
\text { DDP+IL-24 group }\end{array}$ \\
\hline \multicolumn{7}{|c|}{ Weight change of nude mice $(\mathrm{g})$} \\
\hline 0 & $20.2 \pm 0.26$ & $20.1 \pm 0.19$ & $20.0 \pm 0.31$ & $20.1 \pm 0.34$ & $20.0 \pm 0.36$ & $20.0 \pm 0.44$ \\
\hline 3 & $20.6 \pm 0.23$ & $20.5 \pm 0.15$ & $19.8 \pm 0.30$ & $21.2 \pm 0.28$ & $19.6 \pm 0.36$ & $19.7 \pm 0.29$ \\
\hline 6 & $21.4 \pm 0.29$ & $21.4 \pm 0.22$ & $19.4 \pm 0.57$ & $20.7 \pm 0.32$ & $18.1 \pm 1.20$ & $19.2 \pm 0.46$ \\
\hline 9 & $21.9 \pm 0.43$ & $22.2 \pm 0.26$ & $19.2 \pm 0.73$ & $20.7 \pm 0.27$ & $16.7 \pm 1.53$ & $19.0 \pm 0.68$ \\
\hline 12 & $22.5 \pm 0.48$ & $22.8 \pm 0.31$ & $18.8 \pm 0.48$ & $20.5 \pm 0.20$ & $15.9 \pm 1.02$ & $18.6 \pm 0.72$ \\
\hline 15 & $23.3 \pm 0.55$ & $23.7 \pm 0.42$ & $18.4 \pm 0.47$ & $21.1 \pm 0.39$ & $15.6 \pm 0.93$ & $18.2 \pm 0.79$ \\
\hline 18 & $24.0 \pm 0.74$ & $24.4 \pm 0.34$ & $18.4 \pm 0.52$ & $21.2 \pm 0.27$ & $15.3 \pm 0.88$ & $18.6 \pm 0.72$ \\
\hline 21 & $24.6 \pm 0.63$ & $24.9 \pm 0.27$ & $18.3 \pm 0.66$ & $21.3 \pm 0.31$ & $15.6 \pm 1.03$ & $18.5 \pm 0.59$ \\
\hline 24 & $24.9 \pm 0.73$ & $25.1 \pm 0.98$ & $18.1 \pm 0.35$ & $22.0 \pm 0.35$ & $17.1 \pm 0.78$ & $18.6 \pm 0.41$ \\
\hline 27 & $25.3 \pm 0.40$ & $24.9 \pm 1.13$ & $18.2 \pm 1.04$ & $20.9 \pm 0.20$ & $16.9 \pm 0.68$ & $18.8 \pm 0.32$ \\
\hline \multicolumn{7}{|c|}{ Tumor volume change $\left(\mathrm{mm}^{3}\right)$} \\
\hline 0 & $189.5 \pm 47.8$ & $210.5 \pm 95.9$ & $176.9 \pm 100.6$ & $200.1 \pm 79.4$ & $236.1 \pm 135.6$ & $216.1 \pm 54.0$ \\
\hline 3 & $446.3 \pm 73.8$ & $453.8 \pm 125.3$ & $319.9 \pm 73.8$ & $172.9 \pm 63.0$ & $354.0 \pm 105.8$ & $216.3 \pm 74.6$ \\
\hline 6 & $684.1 \pm 95.8$ & $710.7 \pm 139.4$ & $559.2 \pm 104.8$ & $175.0 \pm 47.4$ & $462.3 \pm 103.8$ & $298.2 \pm 42.7$ \\
\hline 9 & $914.6 \pm 131.7$ & $1,015.8 \pm 191.4$ & $581.6 \pm 90.0$ & $199.1 \pm 50.6$ & $573.3 \pm 125.8$ & $319.2 \pm 37.8$ \\
\hline 12 & $1,353.3 \pm 324.9$ & $1,335.0 \pm 347.1$ & $540.8 \pm 108.3$ & $328.8 \pm 45.3$ & $702.2 \pm 148.5$ & $355.8 \pm 47.2$ \\
\hline 15 & $1,532.0 \pm 230.6$ & $1,738.3 \pm 164.0$ & $710.6 \pm 241.1$ & $452.2 \pm 41.4$ & $698.7 \pm 190.3$ & $367.3 \pm 125.0$ \\
\hline 18 & $2,020.0 \pm 213.2$ & $1,940.5 \pm 336.2$ & $1,075.1 \pm 402.9$ & $529.2 \pm 56.0$ & $941.3 \pm 271.1$ & $360.1 \pm 116.2$ \\
\hline 21 & $2,185.5 \pm 262.3$ & $2,147.1 \pm 214.2$ & $1,131.6 \pm 422.3$ & $582.7 \pm 52.8$ & $1,031.5 \pm 161.9$ & $335.6 \pm 117.3$ \\
\hline 24 & $2,609.1 \pm 144.7$ & $2,655.0 \pm 171.1$ & $1,277.9 \pm 455.4$ & $616.9 \pm 28.5$ & $1,201.3 \pm 253.1$ & $381.4 \pm 114.9$ \\
\hline 27 & $2,978.3 \pm 68.6$ & $3,007 \pm 235.4$ & $1,906.3 \pm 514.3$ & $613.1 \pm 44.5$ & $1,446.1 \pm 675.9$ & $281.4 \pm 114.9$ \\
\hline
\end{tabular}

After Mauchly's test of sphericity and Greenhouse-Geisser, Huynh-Feldt or Lower-bound calibrations, t-tests with Bonferroni corrections were performed. There were significant differences between groups (all P-values $<0.05$ ) except between the control group and empty vector group, between the IL-24 group and half-dose DDP+IL-24, and between full-dose and half-dose DDP groups (P>0.05). There were significant differences between groups (all P-values $<0.05$ ) except between the control group and empty vector group, and between the half-dose DDP and half-dose DDP+IL-24 groups $(\mathrm{P}>0.05)$.

Immunohistochemical detection of CD34, specimen preparation and immunohistochemical staining procedures were performed using an SP kit according to the manufacturer's instructions. Subsequently, areas rich in vasculature tissue (known as hot spots) were identified by visualization of the highest density of yellow-brown areas in whole sections under a light microscope (magnification, x100). MVD was calculated at higher magnification microscopy (x400) using the following criteria (10): any brown-stained endothelial cells or cell clusters were recorded as a separate vessel, with clear demarcation between all the vessels. MVD was calculated as the average number of microvessels in five randomly selected fields of vision.

Western blot analysis. Analysis of PDGF-B protein levels in tumor tissue was performed using WesternBreeze ${ }^{\circledR}$ Chemiluminescent kit (Life Technologies, Grand Island, NY, USA) according to the manufacturer's instructions. Tumor tissue protein was extracted, and the concentrations were determined. After SDS-PAGE electrophoresis, it was transferred to a membrane, and immune response and exposure were determined (anti-mouse PDGF-B primary antibody; BioWorld
Products, Visalia, CA, USA). The analysis of experimental results was performed using Quantity One image analysis software.

Statistical methods. SPSS 16.0 statistical software was used for statistical analysis. Multi-group comparisons were performed using ANOVA after the test of normality. t-tests were used for comparisons between two groups. Bonferroni corrections were used if necessary. Non-normal data were compared using SNK tests. Data are presented as means \pm SD. A P-value $<0.05$ was considered to indicate a statistical significant result.

\section{Results}

Inhibition of tumor growth and weight loss of nude mice. Mice in the cisplatin groups (full-dose and half-dose) exhibited poor mental state and reduced feeding. The overall status of mice in the IL-24 and half-dose DDP+IL-24 groups was better than that in the DDP full-dose group. There were no adverse changes in the mental status and feeding habits of nude mice in the IL-24 group (data not shown). 

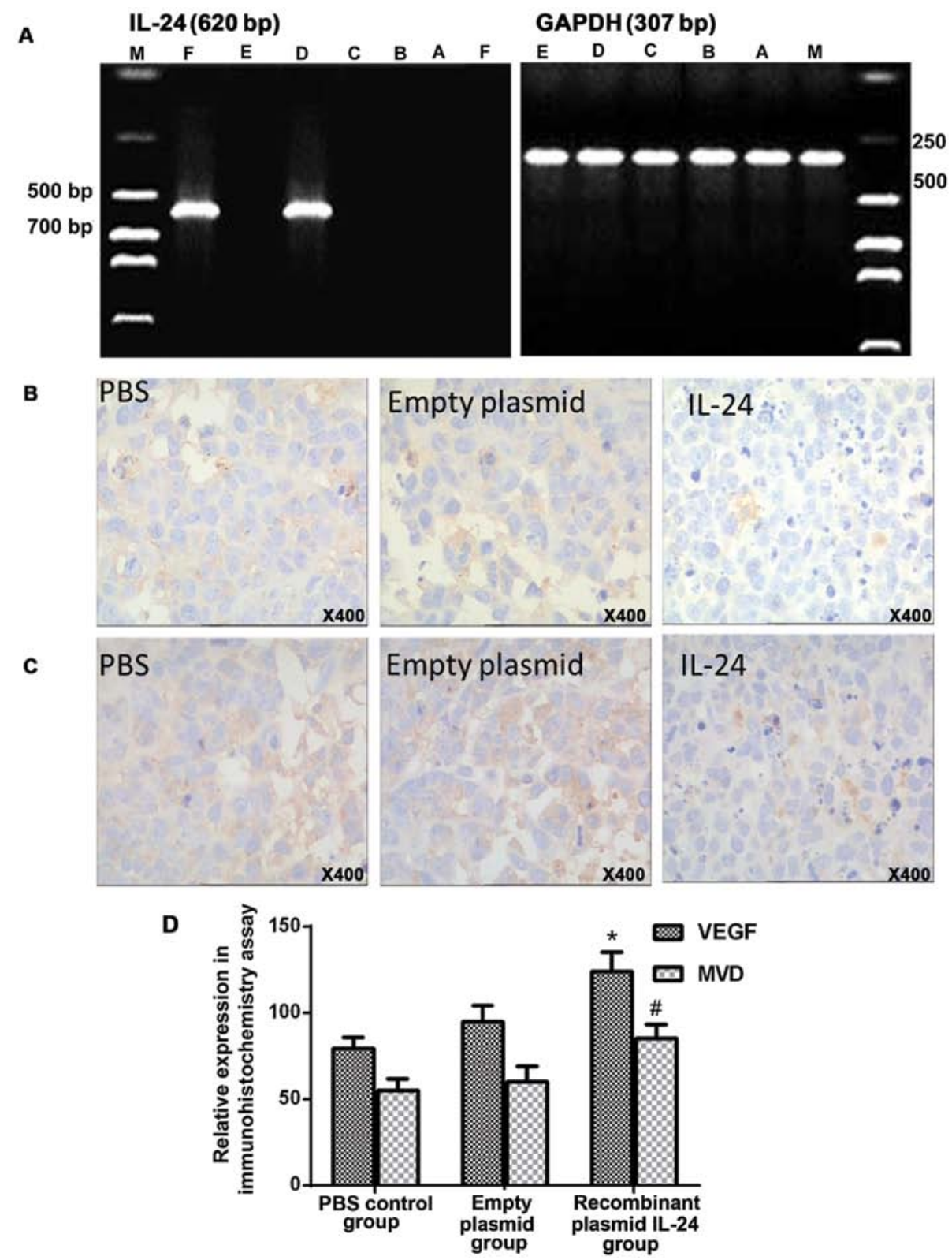

Figure 1. (A) Expression of IL-24 in the xenografts. Lane M, DNA marker; lane A, control group; lane B, empty plasmid group; lane C, half-dose DDP group; lane D, IL-24 group; lane E, full-dose DDP group; lane F, half-dose DDP+IL-24 group. Immunohistochemical detection of expression of (B) VEGF (magnification, $\mathrm{x} 400$ ) and (C) CD34 (magnification, $\mathrm{x} 400$ ). (D) Comparison of intensity scores of panel B and C. ${ }^{* *} \mathrm{P}<0.05$ compared with corresponding control groups.

The changes in tumor volumes after the different interventions are shown in Table I. The tumor volumes were subjected to two-factor analysis of variance. Results of Mauchly's test of sphericity did not support the 'spherical symmetry' hypothesis $(\mathrm{P}<0.001)$; therefore, the coefficient was corrected using Greenhouse-Geisser, Huynh-Feldt and Lower-bound calibrations, and the test results showed statistical significant $\mathrm{P}<0.01$ after calibration. Compared to the control groups (PBS and empty vector), tumor growth was significantly reduced following treatment with cisplatin and/or recombinant IL-24 plasmid. These effects were more marked following treatment with the IL-24 recombinant plasmid and the combined therapy, which mediated almost complete inhibition of tumor growth. The difference was statistically significant at different time-points within the same group $(\mathrm{P}<0.001)$ as well as among different groups $(\mathrm{P}<0.001)$, and there were interaction effects between time and treatment. There were significant differences between groups (all P-values <0.05) except between the control group and empty vector group, between the IL-24 group and half-dose DDP+IL-24, and between the full-dose and half-dose DDP groups $(\mathrm{P}>0.05)$.

Tumor growth was faster in the two control groups, leading to overall weight gain. Cisplatin is associated with serious side-effects. The weight changes of nude mice after intervention are also shown in Table I. Mauchly's test of sphericity was performed and weight changes in the nude mice also refused the'spherical symmetry' hypothesis. The difference was statistically significant at different time-points within the same group $(\mathrm{P}<0.001)$ as well as among different groups $(\mathrm{P}<0.001)$, and there were interaction effects between time and treatment. Results of t-test with Bonferroni corrections revealed that, there were significant differences between groups (all 

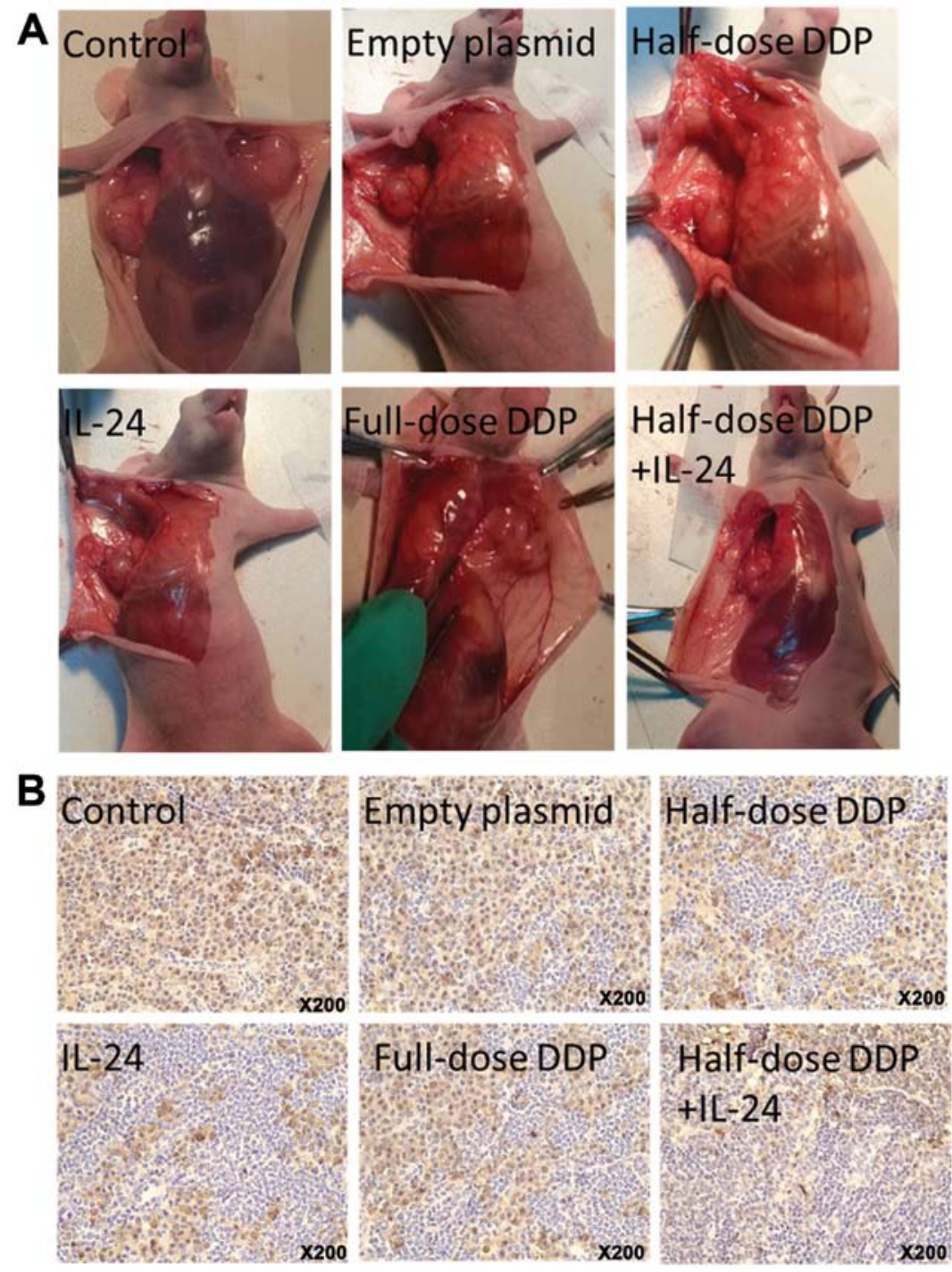

Figure 2. (A) Gross anatomy of mice showing axilla tumor metastasis. (B) Lymph node metastasis marked by cytokeratin (P-CK, magnification, x200).

P-values <0.05) except between the control group and empty vector group, and between the half-dose DDP and half-dose DDP+IL-24 groups $(\mathrm{P}>0.05)$.

IL-24 overexpression increases the expression of VEGF and CD34 in the tumor tissues. Fig. 1A shows expression of IL-24 using RT-PCR in xenograft tumors of the control group, empty plasmid group, half-dose DDP group, IL-24 group, full-dose DDP group, and half-dose DDP+IL-24 group. IL-24 was expressed in the IL-24 group and half-dose DDP+IL-24 group. IHC assays were performed to detect VEGF and CD34 expression in xenograft tumors of the control, empty plasmid and IL-24 groups (Fig. 1B and C). Fig. 1D revealed that there were significant differences in VEGF and CD34 expression between the control group and IL-24 group $(\mathrm{P}<0.001$ and $\mathrm{P}=0.001$, respectively).

Tumor lymph node metastasis. Visible tumor metastatic lymph node was found near the axilla, beside abdominal aortas and in the neck and groin (Fig. 2A). Lymph node metastasis results in the IL-24, cisplatin, combined therapy, empty plasmid and control group mice were $1 / 6,4 / 6,1 / 6,5 / 6$ and $5 / 6$, respectively.
Lymph node metastasis was significantly different between the five groups $\left(\chi^{2}=11.25, P=0.024\right)$. Lymph node metastasis was significantly reduced in the IL-24 and half-dose DDP+IL-24 groups compared with the other groups $(\mathrm{P}<0.05)$.

As a marker of tumor cell infiltration, $\mathrm{P}-\mathrm{CK}$ expression was detected using IHC as brown or tan granules in the cytoplasm of cancer cells (Fig. 2B). Cancer cells in the control and empty plasmid groups were typically observed as hyperchromatic cells with a large nucleus. Tumor cells were arranged in clusters or cords; the volume of tumor cells was large with visible mitotic phase, while the lymphocytes and neutrophils were less. HeLa cell invasion was significantly lower in the IL-24 group compared with that of the control groups. The majority of the infiltrate in the half-dose DDP+IL-24 group comprised lymphocytes and neutrophils, while only minor infiltration and necrosis, larger cells, loose cytoplasm and marked nuclear staining were observed. HeLa cell infiltration was less marked in the cisplatin group, although large nuclei were still observed.

VEGF-C and VEGFR-3 protein expression as markers of lymphangiogenesis. Expression of VEGF-C and VEGFR-3 


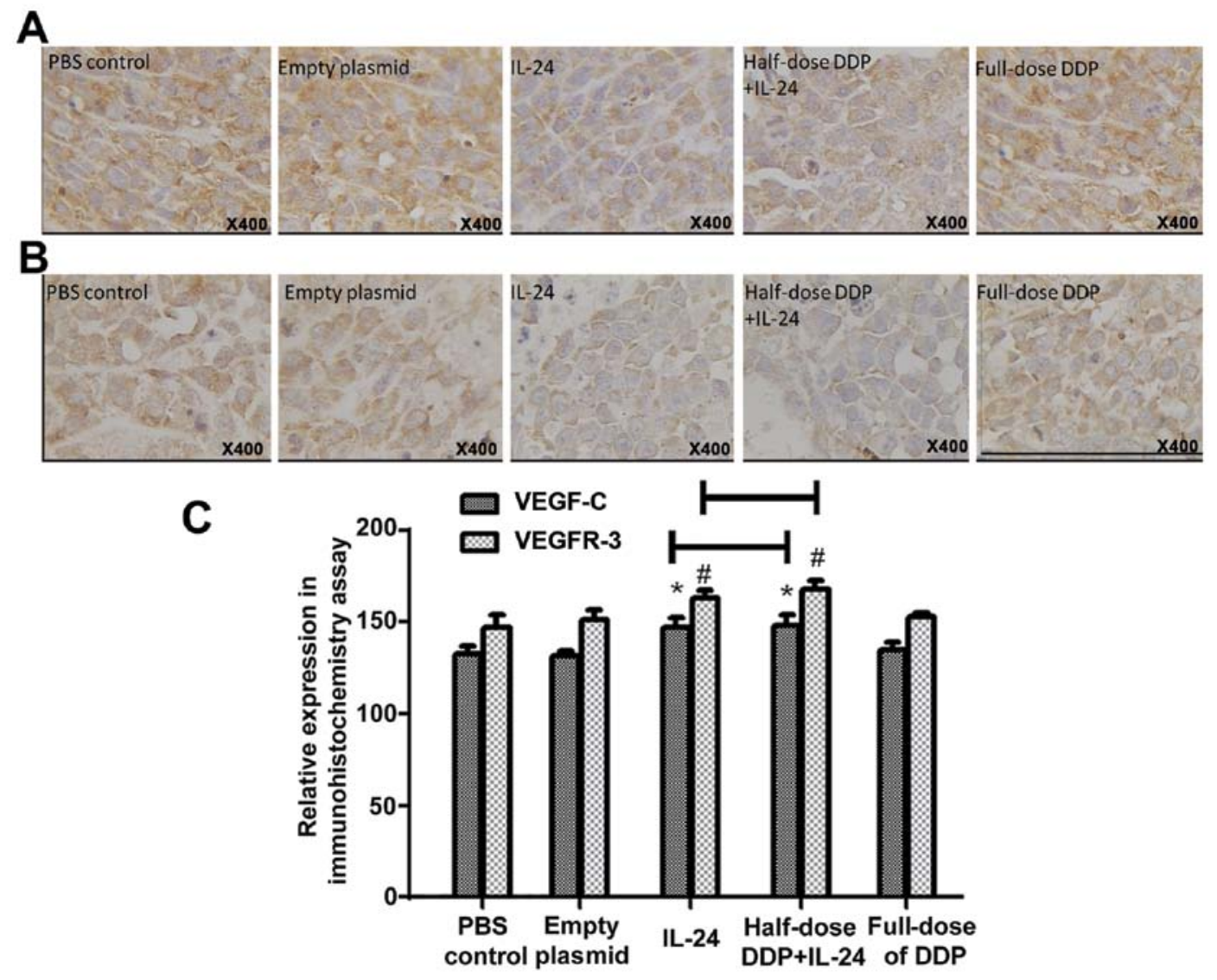

Figure 3. Immunohistochemical staining of (A) VEGF-C (magnification, $\mathrm{x} 400$ ) and (B) VEGFR-3 (magnification, $\mathrm{x} 400$ ). (C) Comparison of the intensity scores. ${ }^{*, \#} \mathrm{P}<0.05$ compared with the corresponding control groups.

was predominantly detected in the cytoplasm of the cancer cells as brownish yellow granules (Fig. 3A and B). Univariate analysis of variance indicated statistically significant differences in the average grayscale values of VEGF-C-positive cells $(\mathrm{F}=17.49, \mathrm{P}<0.001)$ and of VEGFR-3-positive cells $(\mathrm{F}=19.23, \mathrm{P}<0.001)$ between the five groups. The average grayscale values of VEGF-C and VEGFR-3 in the IL-24 and half-dose DDP+IL-24 groups were significantly higher than those in the full-dose DDP, control and empty plasmid groups $(\mathrm{P}<0.001)$, while there were no statistically significant differences between the latter three groups $(\mathrm{P}>0.05)$ (Fig. 3C).

Western blotting and immunohistochemical detection of PDGF-B protein expression. PDGF-B protein was detected in the xenografts of each group by western blotting. Immunohistochemical analysis showed high expression of PDGF-B in the tumor cells in the PBS group, empty plasmid group, DDP group and half-dose DDP group, with no significant differences between the four groups $(P>0.05)$. The levels were significantly reduced in the IL-24 and halfdose DDP+IL-24 groups, compared with that in the other four groups $(\mathrm{P}<0.001)$ (Fig. 4).

\section{Discussion}

Cisplatin is a commonly used chemotherapeutic agent for cervical cancer. However, the development of resistance and serious side-effects limit the efficacy of this approach.
Consequently, the identification of viable adjunct therapies and dosing regimens that increase the sensitivity of tumor cells to the antitumor effects of cisplatin are a current focus of research, with the aim of reducing the effective dose of cisplatin and avoiding its side-effects.

The antitumor activity of IL-24 has been demonstrated in a number of cancers and has shown efficacy in clinical trials for metastatic melanoma (11). Our previous study showed that recombinant IL-24 expressed from the pDC316-hIL-24 vector in combination with cisplatin inhibits the growth of xenografted cervical cancer cell growth in a nude mouse model (7). In the present study, our results obtained using the same animal model showed that recombinant IL-24 acts synergistically with cisplatin to inhibit tumor growth and angiogenesis by downregulation of VEGF, VEGF-C and PDGF-B expression.

Tumor angiogenesis plays an important role in the processes of tumor growth, invasion and metastasis. Angiogenesis depends mainly on the equilibrium between vascular endothelial cell proliferation and inhibitory factors. VEGF, which has a direct effect on vascular endothelial cell growth and vascular permeability, is expressed at high levels in tumor tissue (12). Lymph node metastasis is an important mechanism of cervical cancer invasion and is also an important factor affecting the prognosis of patients. Recent evidence has shown that PDGF, VEGF-C and its receptor VEGFR-3 are closely correlated with angiogenesis, lymphangiogenesis and metastasis in a variety of human malignancies. PDGF-B and its receptor are overexpressed in many human malignant 


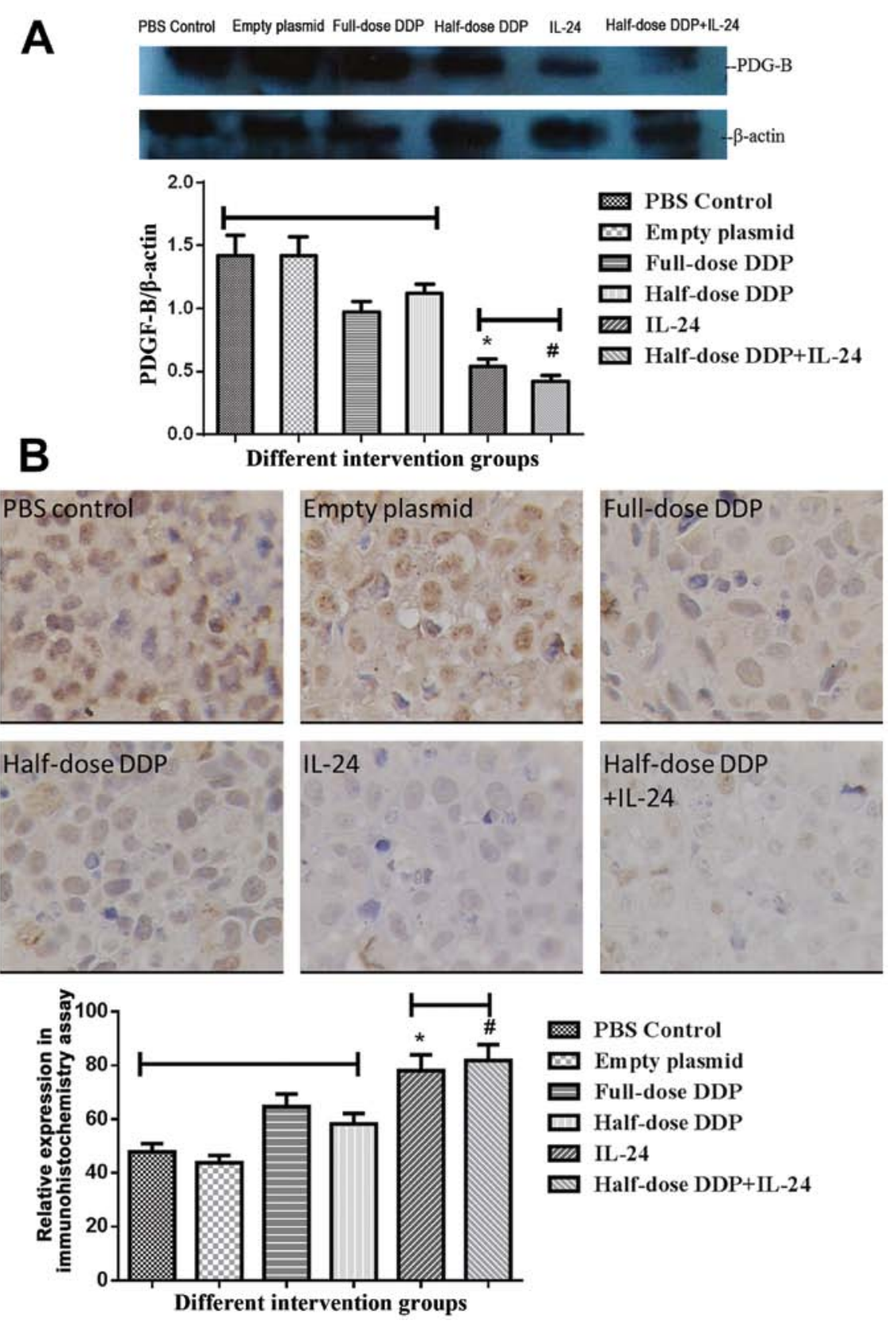

Figure 4. Detection of PDGF-B expression by (A) western blotting and (B) immunohistochemical analyses. Comparisons of the grayscale values or the intensity scores are also shown. ${ }^{*}, \mathrm{P}<0.05$ compared with the corresponding control group.

tumors, and its expression level is correlated with tumor malignancy (13). Donnem et al (14) showed that suppression of PDGF-B expression significantly reduced growth and promoted the apoptosis of lung cancer cells in vitro. Expression of PDGF-B and VEGFR3 is significantly correlated with lymph node metastasis and prognosis of small cell lung cancer (15). Cao (16) reported that PDGF-B transfection caused mouse fibroblast cells to induce lymphangiogenesis and promote regional lymph node metastasis. Furthermore, PDGFR-B has been shown to be specifically expressed at high levels in cervical lesions (17) and correlated with vascular density. Sano et al (18) reported that PDGF-B induces endothelial cell proliferation, migration and formation of tube-like structure blood vessels. Overexpression of PDFG is related to tumor microvessel density in many tumor cells including oral squamous cell carcinoma (19), pancreatic carcinoma (20) or early colon cancer. Furthermore, in the presence of low VEGF expression, the activity of PDGF in infiltrating cells is strongly correlated with MVD (21).

Studies have shown that the interaction between VEGF-C and VEGFR-3 plays a critical role in the growth and survival of lymphatic endothelial cells (22) and promotes tumor lymphangiogenesis and lymphatic metastasis $(23,24)$. Mandriota et al (25) confirmed that VEGF-C and VEGFR-3 promote lymphangiogenesis in a tumor animal model, causing lymphatic invasion and lymph node metastasis. Many studies in human solid tumors such as breast, lung, pancreatic, colon, gastric, esophageal and oral cancers have also identified the importance of the VEGF-C/VEGFR-3 interaction in lymph node metastasis of malignant tumors (26-31). Furthermore, 
Skobe et al (32) reported that VEGF-C induces the formation of new lymphatic vessels and enhances lymphatic metastasis. In a study of VEGF-C expression in cervical cancer and its relationship with the clinical pathological characteristics of cervical cancer, Harshimoto et al (33) reported that VEGF-C expression was significantly higher in cancers with deeper stromal invasion and lymph node metastasis.

In the present study, the expression of VEGF and MVD was lower in the recombinant IL-24 group compared with that in the control and empty plasmid groups, indicating a reduction in tumor angiogenesis. This resulted in tumor blood supply decrease, which further inhibited tumor growth. Furthermore, VEGF-C and VEGFR-3 expression was increased in the recombinant IL-24 and combined treatment groups compared with the other groups, indicating that IL-24 downregulates the expression of these molecules. Lymph node metastasis was reduced in the IL-24 and combined treatment groups compared with the other groups, suggesting that IL-24 plays a role in inhibiting lymphatic metastasis of transplanted tumor by downregulating the expression of VEGF-C. PDGF-B was highly expressed in the PBS control and empty plasmid groups, whereas after the IL-24 intervention, and especially after intervention with cisplatin, PDGF-B expression was significantly decreased. Tumor volume and tumor inhibition were also significantly reduced in the intervention group. These observations suggest that increased PDGF-B has a positive correlation with tumor invasion and metastasis. Thus, inhibition of the expression of PDGF-B family factors and other angiogenic factors is implicated as one of the mechanisms by which IL-24 inhibits tumor growth and metastasis.

IL-24 had no obvious side-effects in terms of animal weight loss in this experimental animal model, which is consistent with the absence of reports of IL-24 toxicity to normal cells (34). It can also reduce the chemotherapy drug dosage and improve the quality of life of patients.

In summary, the results of the present study showed that recombinant IL-24 acts synergistically with cisplatin to inhibit tumor growth and angiogenesis. Our data indicate that these effects are mediated by downregulation of VEGF, VEGF-C and PDGF-B expression. Thus, IL-24 is implicated as a potential adjunct therapy to enhance tumor chemosensitivity to cisplatin

\section{References}

1. Ferlay J, Soerjomataram I, Dikshit R, et al: Cancer incidence and mortality worldwide: Sources, methods and major patterns in GLOBOCAN 2012. Int J Cancer 36: E359-E386, 2014.

2. Wang Z, Kong D, Banerjee S, et al: Down-regulation of platelet-derived growth factor-D inhibits cell growth and angiogenesis through inactivation of Notch-1 and nuclear factorkappaB signaling. Cancer Res 67: 11377-11385, 2007.

3. Ramesh R, Ito I, Gopalan B, Saito Y, Mhashilkar AM and Chada S: Ectopic production of MDA-7/IL-24 inhibits invasion and migration of human lung cancer cells. Mol Ther 9: 510-518, 2004.

4. Gopalan B, Shanker M, Chada S and Ramesh R: MDA-7/IL-24 suppresses human ovarian carcinoma growth in vitro and in vivo. Mol Cancer 6: 11, 2007.

5. Sarkar D, Su ZZ, Vozhilla N, Park ES, Gupta P and Fisher PB: Dual cancer-specific targeting strategy cures primary and distant breast carcinomas in nude mice. Proc Natl Acad Sci USA 102: 14034-14039, 2005.
6. Gupta P, Su ZZ, Lebedeva IV, et al: mda-7/IL-24: multifunctional cancer-specific apoptosis-inducing cytokine. Pharmacol Ther 111: 596-628, 2006.

7. Li L, Wang ZX and Wang ZH: Combination of IL-24 and cisplatin inhibits cervical cancer growth in a xenograft nude mice model. Asian Pac J Cancer Prev 12: 3293-3298, 2011.

8. Bresalier RS, Ho SB, Schoeppner HL, et al: Enhanced sialylation of mucin-associated carbohydrate structures in human colon cancer metastasis. Gastroenterology 110: 1354-1367, 1996.

9. Traweek ST, Kandalaft PL, Mehta P and Battifora H: The human hematopoietic progenitor cell antigen (CD34) in vascular neoplasia. Am J Clin Pathol 96: 25-31, 1991.

10. Weidner N: Current pathologic methods for measuring intratumoral microvessel density within breast carcinoma and other solid tumors. Breast Cancer Res Treat 36: 169-180, 1995.

11. Lee HN, Lee KH, Lee DW, Lee YS, Park EK and Park JS: Weekly cisplatin therapy compared with triweekly combination chemotherapy as concurrent adjuvant chemoradiation therapy after radical hysterectomy for cervical cancer. Int J Gynecol Cancer 21: 128-136, 2011.

12. Ishikawa M, Kitayama J, Kazama S and Nagawa H: Expression of vascular endothelial growth factor $\mathrm{C}$ and $\mathrm{D}$ (VEGF-C and -D) is an important risk factor for lymphatic metastasis in undifferentiated early gastric carcinoma. Jpn J Clin Oncol 33: 21-27, 2003.

13. Nakamura Y, Tanaka F, Yoshikawa Y, et al: PDGF-BB is a novel prognostic factor in colorectal cancer. Ann Surg Oncol 15: 2129-2136, 2008.

14. Donnem T, Al-Saad S, Al-Shibli K, Andersen S, Busund LT and Bremnes RM: Prognostic impact of platelet-derived growth factors in non-small cell lung cancer tumor and stromal cells. J Thorac Oncol 3: 963-970, 2008.

15. Donnem T, Al-Saad S, Al-Shibli K, Busund LT and Bremnes RM: Co-expression of PDGF-B and VEGFR-3 strongly correlates with lymph node metastasis and poor survival in non-small-cell lung cancer. Ann Oncol 21: 223-231, 2010.

16. Cao Y: Direct role of PDGF-BB in lymphangiogenesis and lymphatic metastasis. Cell Cycle 4: 228-230, 2005.

17. Mayer TJ, Frauenhoffer EE and Meyers AC: Expression of epidermal growth factor and platelet-derived growth factor receptors during cervical carcinogenesis. In Vitro Cell Dev Biol Anim 36: 667-676, 2000.

18. Sano H, Ueda Y, Takakura N, et al: Blockade of plateletderived growth factor receptor-beta pathway induces apoptosis of vascular endothelial cells and disrupts glomerular capillary formation in neonatal mice. Am J Pathol 161: 135-143, 2002.

19. Li C, Shintani S, Terakado N, et al: Microvessel density and expression of vascular endothelial growth factor, basic fibroblast growth factor, and platelet-derived endothelial growth factor in oral squamous cell carcinomas. Int J Oral Maxillofac Surg 34: $559-565,2005$.

20. Fujimoto K, Hosotani R, Wada M, et al: Expression of two angiogenic factors, vascular endothelial growth factor and platelet-derived endothelial cell growth factor in human pancreatic cancer, and its relationship to angiogenesis. Eur J Cancer 34: 1439-1447, 1998.

21. Takahashi Y, Bucana CD, Liu W, et al: Platelet-derived endothelial cell growth factor in human colon cancer angiogenesis: role of infiltrating cells. J Natl Cancer Inst 88: 1146-1151, 1996.

22. Makinen T, Veikkola T, Mustjoki S, et al: Isolated lymphatic endothelial cells transduce growth, survival and migratory signals via the VEGF-C/D receptor VEGFR-3. EMBO J 20: 4762-4773, 2001.

23. Zu X, Tang Z, Li Y, Gao N, Ding J and Qi L: Vascular endothelial growth factor-C expression in bladder transitional cell cancer and its relationship to lymph node metastasis. BJU Int 98: 1090-1093, 2006.

24. Matsumoto M, Natsugoe S, Okumura H, et al: Overexpression of vascular endothelial growth factor-C correlates with lymph node micrometastasis in submucosal esophageal cancer. J Gastrointest Surg 10: 1016-1022, 2006.

25. Mandriota SJ, Jussila L, Jeltsch M, et al: Vascular endothelial growth factor-C-mediated lymphangiogenesis promotes tumour metastasis. EMBO J 20: 672-682, 2001.

26. Tsurusaki T, Kanda S, Sakai H, et al: Vascular endothelial growth factor-C expression in human prostatic carcinoma and its relationship to lymph node metastasis. Br J Cancer 80: 309-313, 1999. 
27. Valtola R, Salven P, Heikkila P, et al: VEGFR-3 and its ligand VEGF-C are associated with angiogenesis in breast cancer. Am J Pathol 154: 1381-1390, 1999.

28. Kajita T, Ohta Y, Kimura K, et al: The expression of vascular endothelial growth factor $\mathrm{C}$ and its receptors in non-small cell lung cancer. Br J Cancer 85: 255-260, 2001.

29. Yonemura Y, Fushida S, Bando E, et al: Lymphangiogenesis and the vascular endothelial growth factor receptor (VEGFR)-3 in gastric cancer. Eur J Cancer 37: 918-923, 2001.

30. Kitadai Y, Amioka T, Haruma K, et al: Clinicopathological significance of vascular endothelial growth factor (VEGF)-C in human esophageal squamous cell carcinomas. Int J Cancer 93: 662-666, 2001.
31. Saaristo A, Partanen TA, Arola J, et al: Vascular endothelial growth factor-C and its receptor VEGFR-3 in the nasal mucosa and in nasopharyngeal tumors. Am J Pathol 157: 7-14, 2000.

32. Skobe M, Hawighorst T, Jackson DG, et al: Induction of tumor lymphangiogenesis by VEGF-C promotes breast cancer metastasis. Nat Med 7: 192-198, 2001.

33. Hashimoto I, Kodama J, Seki N, et al: Vascular endothelial growth factor-C expression and its relationship to pelvic lymph node status in invasive cervical cancer. Br J Cancer 85: 93-97, 2001.

34. Su Z, Emdad L, Sauane M, et al: Unique aspects of $m d a-7 / I L-24$ antitumor bystander activity: establishing a role for secretion of MDA-7/IL-24 protein by normal cells. Oncogene 24: 7552-7566, 2005. 